\title{
When exotic introductions fail: updating invasion beliefs
}

\author{
Daniel K. Heersink · Peter Caley $(\mathbb{D} \cdot$ Dean Paini $\cdot$ Simon C. Barry
}

Received: 19 November 2019/Accepted: 25 November 2019/Published online: 6 December 2019

(C) The Author(s) 2019

\begin{abstract}
Decisions regarding invasive risk of exotic species are often based on species distribution models projected onto the recipient region of interest. Such projections are essentially a measure of prior belief in the ability of an organism to invade. Whilst many decisions are made on the basis of such projections, it is less clear how such prior belief may be empirically modified on the basis of data, in particular introduction events that haven't led to establishment. Here, using the Asian green mussel (Perna viridis) as an example, we illustrate how information on failed introduction attempts may be used to continually update our beliefs in the ability of an organism to invade per introduction, and the underlying habitat suitability for establishment. Our results show that the establishment probability of $P$. viridis per fouled ship visit in the supposedly favourable northern Australian waters are much lower than initially though, and are continuing to decline. A Bayesian interpretation of our results notes the dramatic reduction in our belief of the ability of $P$. viridis to invade in the light of what we estimate to be 100's of fouled vessels per year visiting ports without any persistent populations establishing. Under a hypothetico-deductive approach we would reject the
\end{abstract}

D. K. Heersink · P. Caley $(\square) \cdot$ S. C. Barry

CSIRO Data61, Canberra, Australia

e-mail: Peter.Caley@csiro.au

D. Paini

CSIRO Health and Biosecurity, Canberra, Australia null (prior) species distribution model as being useful, and seek to find a better one that can withstand the challenge of data.

Keywords Invasion - Propagule pressure $\cdot$ Perna viridis $\cdot$ Asian green mussel $\cdot$ Species distribution model $\cdot$ Climate matching

\section{Introduction}

Understanding and predicting the distribution and abundance of an invasive species in a new environment is a fundamental question in invasion ecology, as it is with ecology more generally (Krebs 2009). Species distribution models (SDMs) have increasingly been used for this task-see Elith and Leathwick (2009) for a review of fitting SDMs, and Elith et al. (2010) for issues relating to building SDMs for rangeshifting species. The time and effort required to generate an SDM are minuscule in comparison to those needed to test the resulting projections. Indeed, one could argue the ecological world is being "overrun" by SDMs. Any working ecologist will admit the answers to questions of the distribution and abundance of organisms are often complex and site-specific. Increasingly, in an evidence-based policy environment, biosecurity decisions relating to potentially invasive alien species are made on the basis of SDM 
predictions - which species are at most risk of establishing and spreading following entry. Such predictions are often overlaid on asset layers, as a means of estimating impact, and form the basis of management decisions (e.g. Paini et al. 2018).

We note, however, it is becoming increasingly apparent that SDMs, especially those fitted to climatic data alone, don't reliably project well to new environments (Beale and Lennon 2011). This should come as no surprise, as the problems of projecting statistical models beyond the training data are well known historically. So a priori, we expect that some SDM predictions will be in error-species predicted as able to establish in fact can't, and vice versa. Such underlying uncertainty is often hard to quantify, and rarely presented. In fact, conversely, the graduated graphics of species distribution models give the impression of detailed, precise underlying prediction. The focus to date has generally been on SDMs underpredicting within invaded ranges (e.g. Fitzpatrick et al. 2007), and what may be the underlying cause (e.g. model over-fitting), and possible remedies (Elith et al. 2010). Instances of over-prediction are much less commonly reported on, but note the possibility of some selection bias in operation-studies tend to focus on those species that have already successfully invaded new regions.

Given these underlying problems with SDM predictions, it makes sense to treat them as a measure of prior belief in the ability of an organism to establish. It follows that such beliefs should be updated as additional data come to hand, or alternative methods are applied.

An obvious source of additional data is introduction effort. It is widely accepted that the greater the number of introduction events (propagules), and the number of individuals involved (propagule size), the greater the probability of establishment, for reasons of surviving environmental and/or demographic stochasticity, overcoming Allee effects (if applicable), and having sufficient genetic variation (if needed) to adapt to local conditions and thus become self-sustaining (see Lockwood et al. 2009; Blackburn et al. 2009). Under this premise, establishment is possible, given enough attempts. The exact probability per introduction attempt (or per propagule) is rarely known (or estimated).

This paper addresses how we may update beliefs regarding both habitat suitability and establishment ability in the light of additional knowledge arising from failed introductions. Specifically it explores, how to update our beliefs regarding a species ability to establish using knowledge of introduction failures. The concepts are illustrated via a case study of introductions and establishment success of the Asian green mussel (Perna viridis) into Australia. Two key questions are addressed. First, can $P$. viridis establish in Australia given that it is introduced? Second, if it can establish, what is the likelihood of this happening per introduction event, and cumulatively accounting for all introduction events? These questions are tackled empirically using a hiearchical Bayesian model that incorporates the current approach rate of $P$. viridis fouled vessels into Australia over the last 50 years, with a temperature based species distribution model that reflects current beliefs regarding invasion risk.

\section{Methods}

Background: Asian green mussel establishment risks

During the 1990s, there was increasing concern with human-mediated changes to marine environments, with particular concern as to human-mediated introductions of exotic marine species through shipping traffic (ballast water, hull fouling) and aquaculture (Cariton and Geller 1993; Thresher 1999). Temperate marine habitats appeared particularly prone to colonization of exotic species [though note that Thresher (1999) considered that research had been biased these habitats at the time]. For example, by 1996, Port Phillip Bay was host to 99 introduced marine species, representing $c$. $8 \%$ of all recorded species (Hewitt et al. 2004). At the time, the rate of invasions was thought to be increasing as a result of increased shipping traffic, although changes in sampling effort were also considered a possible explanation, and reported discoveries of species considered non-indigenous peaked in this location in the 1970s (Hewitt et al. 2004) [in contrast to the broader findings of Seebens et al. (2017)]. In response, invasive species threat lists were generated (e.g. Hayes et al. 2005), ballast water management risk assessments undertaken (e.g. Barry et al. 2008), and protocols implemented domestically and internationally. 
The Asian green mussel was identified as a species of invasion concern for Australia on the basis of shipmediated invasion history in other regions, the presence of demonstrated shipping vectors, and its ability to cause harm (Hayes and Sliwa 2003). Native to the Indo-Pacific region, this species has been invasive when introduced outside its native range, displacing local native species (NIMPIS 2014; Baker et al. 2007). Further to its potentially invasive nature of marine ecosystems, $P$. viridis is a serious biofouling pest of hard substrates including vessels and industrial infrastructure (NIMPIS 2014).

Individuals of $P$. viridis have been detected on several vessels during routine vessel inspections since 1999, including evidence of spawning from some of these detections in Australian waters. This has generated concern that the establishment of $P$. viridis in Australia may be imminent.

Using simple matching criteria, $P$. viridis certainly fits the profile of a high risk species-it has a history of invasiveness when introduced outside its native range (Benson et al. 2001; Stafford et al. 2007), and would find the water temperature range across much of northern Australia within its tolerances (Hayes et al. 2008). Indeed, $P$. viridis was rated as one of the ten most likely invaders using a measure of environmental similarity (Hayes et al. 2005). In response to this perceived high risk, considerable resources are spent controlling and screening for this species.

\section{A SDM based on water temperature}

Biological factors affecting the culture of $P$. viridis include, among others, water temperature, water salinity, and nutrient availability (Vakily 1989). The reported 50\% survival temperature tolerance range for $P$. viridis is $10-35{ }^{\circ} \mathrm{C}$, with an optimal range of $26-$ $32{ }^{\circ} \mathrm{C}$ (Sivalingam 1977). The $50 \%$ survival salinity tolerance range for $P$. viridis is reported as $24-80 \mathrm{ppt}$ (Sivalingam 1977), with an optimal range of 27-65 ppt. A wide range of chlorophyll- $\alpha$ levels was deemed suitable for sustaining $P$. viridis culture, with 17$40 \mu \mathrm{g} / \mathrm{l}$ water (Cheong 1982) and 3.5-5.2 $\mu \mathrm{g} / \mathrm{l}$ (Sivalingam 1977) reported as suitable.

Sea surface temperature (SST) is the most common environmental variable used to predict the potential distribution of $P$. viridis (Summerson et al. 2007; Hayes et al. 2008). Whilst a model based on SST can be viewed as simplistic, it forms the basis of our current belief regarding port suitability, hence we take this approach. The potential use of other environmental covariates is addressed in the Discussion.

Twelve months (April 2013-March 2014) of SST levels for the entire globe were sourced from https:// neo.sci.gsfc.nasa.gov on a $0.5^{\circ}$ resolution. These values were then averaged over the 12 months span. To more accurately delineate locations within Australia that are potentially suitable habitat for $P$. viridis, only locations with SST within the optimal range for $P$. viridis were used. Therefore we let

$S S T^{*}=\left\{\begin{array}{ll}S S T, & 26 \leq S S T \leq 32 \\ 0, & \text { otherwise }\end{array}\right.$.

Applying this optimal temperature range reveals the most suitable areas for $P$. viridis in Australia are north of $30^{\circ} \mathrm{S}$ latitude, and realistically north of the Tropic of Capricorn (Fig. 1), similar to the habitable range for proposed by Summerson et al. (2007) and Hayes et al. (2008). Note that Summerson et al. (2007) used a wider temperature range than was used here and Hayes et al. (2008) calculated the potential $P$. viridis range using seasonal averages instead of yearly average temperatures.

In a statistical sense, a model using SST can be used to generate a prior belief $(\psi)$ that a port location is suitable for $P$. viridis. Data to calibrate such a model is available in Baker et al. (2007), who provides a list of known worldwide introductions of $P$. viridis, including the vector. Two of these introductions (Cape Verde, Cook Islands) that were intentional (aquaculture) were unsuccessful and can serve as indicating unsuitable environment ( $y_{i}=0$ cases), particularly given the associated propagule introduction effort. We also include the introduction (spawning event) in Perth (Garden Island) on a naval vessel as a failure (i.e. 0), as during a major spawning event no mussels successfully colonized non-shipping surfaces. Values of SST were calculated for all $n=19$ locations with a known establishment outcome. These locations are: Cape Verde; Cook Islands; Garden Island, Australia; Singapore; Hong Kong; New Caledonia; Trinidad; Tampa, Florida; Fiji; Tonga; Tahiti; Western Samoa; Okinawa, Japan; Taiwan; Honshu, Japan; and Kingston, Jamaica. The establishment of $P$. viridis, $y_{i}$, was then modelled via logistic regression as: 


\section{Optimal Temperature Range}

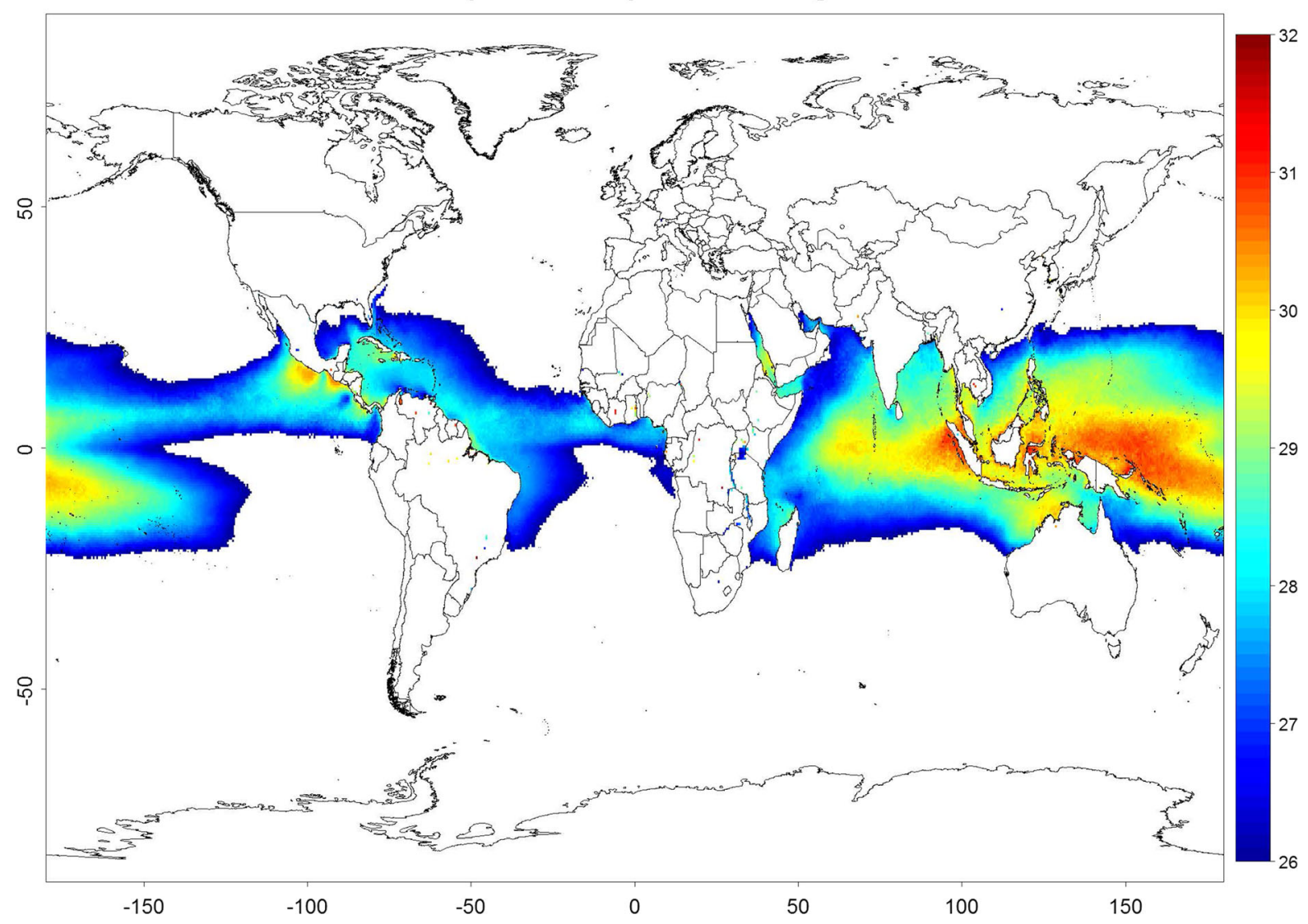

Fig. 1 Optimal sea surface temperature range of $P$. viridis based on satellite data and literature review

$y_{i} \sim \operatorname{Bernoulli}\left(\psi_{i}\right)$, with

$\operatorname{logit}\left(\psi_{i}\right)=\beta_{0}+\beta_{1} S S T_{i}^{*}$.

The predicted suitability $\psi_{i}$ for each Australian port $i$, was then calculated using the mean SST calculated as the mean of the surrounding nine grid cells $\left(S S T_{i}\right)$.

The long-run suitability of Australian ports north of $30^{\circ} \mathrm{S}$ latitude $(n=27)$ was then predicted as

$$
\widehat{\psi}_{i}=\frac{\exp \left(\widehat{\beta}_{0}+\widehat{\beta}_{1} S S T_{i}^{*}\right)}{1+\exp \left(\widehat{\beta}_{0}+\widehat{\beta}_{1} S S T_{i}^{*}\right)} .
$$

We recognise that this is not the most sophisticated model that could be built. However, including available data on salinity and chlorophyll- $\alpha$ levels at the resolution available did not change the distribution materially. We consider the model to be adequate for our purposes, as the predictions are very similar to the models of Summerson et al. (2007) and Hayes et al. (2008).

\section{Estimating introductions}

The rate of introductions (the "approach rate") of $P$. viridis was estimated using available data on biofouling and historical shipping records as the product of fouling rate and shipping volume summed over all possible vessel types (see Data Sources below within Results for details). That is,

$N_{t, i, j}=\sum_{k} q_{t, i, j, k} \times x_{t, i, j, k}$

where $q_{t, i, j, k}$ is the fouling rate/proportion of vessels/substrates of type $k$, from country $j$, arriving in region $i$, in year $t$ and $x_{t, i, j, k}$ is the total number of vessels/substrates of type $k$, from country $j$, arriving in region $i$, in year $t$. 
We assume that the fouling rate is constant across all substrates, countries of origin, and time, as there is not enough resolution in the available fouling data to estimate time, country or substrate dependent values for $q_{t, i, j, k}$, but a sensitivity analysis can be performed to investigate the importance of this parameter. There is some observational evidence (Richard Willan, personal communication) that $P$. viridis will attach on a wide range of substrates. We therefore set $q_{t, i, j, k}=q$.

A simple estimate of $q$ is the ratio of observed fouled vessels arriving to the recipient country to the total volume of international vessels arriving from endemic $P$. viridis waters:

$\widehat{q}=\sum_{t, i, j} N_{t, i, j} / \sum_{t, i, j} x_{t, i, j}$

We use a Poisson regression model to estimate the total number of international vessel arrivals for each of the last 50 years, $x_{t, i, j}$, using country of origin, year, and total imports from the country in Australian dollars (AUD). This approach was made necessary by the lack of shipping data over the time period of interest. The total value of imports by country to each individual state region is not readily available for the last 50 years, so import value to all of Australia was used. The proposed model is thus:

$$
\begin{gathered}
x_{t, j} \sim \text { Poisson }\left(\mu_{t, j}\right), \quad t=1964,1965, \ldots, 2013, \text { with } \\
\log \left(\mu_{t, j}\right)=\beta_{0}+\beta_{j} \text { Country }_{j}+\gamma_{1} t+\gamma_{2} \text { Import }_{t},
\end{gathered}
$$

where Country $_{j}$ is a categorical variable. Predictions for years without shipping data available is then straightforward:

$$
\widehat{x}_{t, j}=\exp \left(\widehat{\beta}_{0}+\widehat{\beta}_{j} \text { Country }_{j}+\widehat{\gamma}_{1} t+\widehat{\gamma}_{2} \text { Import } \$_{t}\right) \text {. }
$$

Let $\delta_{t, i, j}$ be the proportion of all international vessel arrivals that are from country $j$ arriving in year $t$ and in Australian region $i$. If we assume that shipping patterns are relatively constant over the last 50 years, then $\delta_{t, i, j} \approx \delta_{i, j}$. This can be estimated using all available shipping data to get

$$
\begin{aligned}
\widehat{\delta}_{i, j} & =\sum_{t} x_{t, i, j} / \sum_{t, i, j} x_{t, i, j}, \quad \text { and } \\
\widehat{x}_{t, i, j} & =\widehat{\delta}_{i, j} \times \widehat{x}_{t, j} .
\end{aligned}
$$

Finally, the total number of fouled vessels arriving in year $t$ and region $i$ from country $j$ is estimated as

$$
\widehat{N}_{t, i, j}=\widehat{q} \times \widehat{x}_{t, i, j}
$$

\section{Updating invasion beliefs}

A summary of how estimates of the approach rate, habitat suitability and the historical record of interceptions and incursions in Australia can be used to update belief in the invasion parameters of $P$. viridis is as follows. First, prior beliefs for the region-based suitability of ports are derived from the temperaturebased habitat suitability models. This prior belief is updated on the basis of the number of fouled arrivals and the probability of establishment per arrival. We then update estimates of the probability of establishment per arrival, and the overall probability of establishment arising from all arrivals, conditional on the habitat being suitable.

\section{Model specification}

To estimate the probability that $P$. viridis can establish in a recipient region of interest. Let $z$ be an state variable defined as

$z= \begin{cases}0, & \text { if } P . \text { viridiscannot establish in recipient region } \\ 1, & \text { if } P . \text { viridis can establish in recipient region. }\end{cases}$

The probability that $P$. viridis establishment is at all possible in a region is denoted by $\theta$. That is, $P(z=$ 1) $=\theta$ and $P(z=0)=1-\theta$.

We model $z$ as arising from a Bernoulli trial. That is, $z \sim \operatorname{Bernoulli}(\theta)$, with the results from the species distribution model are used to inform the prior specification for $\theta$. For each region we estimate an average value of port suitability $\widehat{\theta}_{i}=\frac{1}{n_{r}} \sum_{r} \widehat{\psi}_{r}$, where the sum is over all ports in the region, $n_{r}$ is the number of ports under consideration in the region, and the $\widehat{\psi}_{r}$ are the prior beliefs of suitability based on mean SST.

The value of $\theta$ is informed (i.e. "learning occurs") as fouled vessels arrive in a region, depending on the outcome. If an establishment occurs, then $z=1$ - that 
is, the habitat is suitable for establishment and remains that way (in the absence of habitat modification). If no establishment has occurred after $N$ introductions we can update the probability that $z=1$ by multiplying by the probability that all of those entries resulted in no establishment. Formally, let $p$ be the probability that a fouled entry results in an establishment and assume, a priori, that it has a $\operatorname{Beta}(\alpha, \beta)$ distribution. Then, if after $N$ introductions there is no establishment, the joint posterior distribution (as denoted by square brackets) for recipient environment suitability and the establishment probability per introduction is defined by:

$$
\begin{aligned}
& {[z=0, p \mid N] \propto(1-\theta)[p], \quad \text { and }} \\
& {[z=1, p \mid N] \propto \theta(1-p)^{N}[p] .}
\end{aligned}
$$

The marginal densities for suitability states $(z=0$ and $z=1)$ are found by integrating with respect to $p$ and normalizing, noting the conditional distribution for $z=0$ doesn't depend on $p$. Furthermore, conditional on the habitat being suitable, we can estimate the probability of an introduction resulting in establishment as

$$
[p \mid z=1, N]=\operatorname{Beta}(p ; \alpha, \beta+N) .
$$

From a risk management perspective, we then wish to estimate the overall probability $(\pi)$ of $P$. viridis establishing in a recipient region, given the approach rate of fouled vessels and the suitability of the port habitat. If $z=0$, then this probability is also zero. If $z=1$, we can estimate the conditional probability of establishment of $P$. viridis in the recipient region as a result of potentially multiple (say $N$ ) fouled vessel entries. With reasonable estimates of the approach rate and fouling rate, this can be estimated as:

$$
\begin{aligned}
P(\text { Establishment } \mid z=1) & =P(\text { Establishment } \mid N \text { arrivals }) \\
& =1-P(\text { All } N \text { arrivals fail }) \\
& =1-(P(\text { Fouled arrival fails }))^{N} \\
& =1-(1-\hat{p})^{N}=\pi^{c},
\end{aligned}
$$

where $\hat{p}$ is the probability of establishment per individual fouled vessel arrival in that region assuming the habitat is suitable. The superscript for $\pi$ in Eq. 10 indicates it is conditional on the recipient habitat being suitable. The value of $p$ is assumed to be constant for each port within a recipient region. This is a conservative assumption as this aggregates all establishment events in a region into one data point for each year and region.

\section{Overall risk}

The final, unconditional probability of establishment is then defined as

$P($ Establishment $)=P($ Establishment $\mid z=1) \times P(z=1)$.

Analyses were undertaken separately for each state region considered to be at risk of $P$. viridis invasion.

Computations were undertaken using the R statistical software (R Core Team 2018).

\section{Results}

Data sources: shipping volumes and fouling prevalence

The fouling rate $(q)$ was estimated using naval vessel movement and inspection data sourced from the Australian Defence Science and Technology Organisation. The quality and thoroughness of naval vessel inspections provides an ideal sample of total international vessel movement with which to estimate $q$. During the time frame in which the navy has inspected its vessels for $P$. viridis (December 2007 to present), there have been eleven positive identifications of $P$. viridis ( $\bar{x}=60 \pm 30$ individuals of mixed age). That same time frame has seen 306 naval vessels travel from southeast Asia to Australia. For this study, all southeast Asian countries the navy visits within the native range of $P$. viridis were used. These countries are Brunei, China, India, Indonesia, Japan, Malaysia, Philippines, Singapore, Sri Lanka, Thailand and Vietnam.

The port history of each infested naval vessel indicates a stop in Singapore of 4-10 days within the previous 6 months. There were a total of 120 vessels arriving in Australia from Singapore. The proportion of infested vessels to total vessels provides an estimate of the fouling rate of $P$. viridis. If all vessels returning to Australia from southeast Asia are utilised, this estimated fouling rate is $\widehat{q}_{1}=0.0359$. If only those vessels returning to Australia from Singapore are 
considered, the estimated fouling rate is $\widehat{q}_{2}=0.0917$. As it is unknown where these naval vessels became infested with $P$. viridis, we use the mean of these two estimates as the estimate of fouling rate: $\widehat{q}=\frac{\widehat{q}_{1}+\widehat{q}_{2}}{2}=0.0638$.

To estimate the number of international vessel arrivals ( $\widehat{x}_{t, j}$ in Eq. 7), the historical value of imports by country was sourced from Australian Bureau of Statistics (ABS) Yearbooks from 1963-1964 to 2012-2013. Historical international vessel arrivals for 1998 to 2013 were sourced from Lloyds List Intelligence (LLI) via the Bureau of Infrastructure, Transport and Regional Economics (BITRE). Model Eq. 6 was then fit and estimates calculated for all years 1964-2013.

The estimated number of international vessel arrivals values show an exponential growth pattern, with little change in the relative contribution for different countries other than China, with growth in vessel numbers rising disproportionately over the past 15 years.

\section{Approach rate and prior habitat suitability}

The estimated number of fouled vessels arriving in each state region, $\widehat{N}_{t, i}$, is substantial (from Eq. 9), and this has been the case for several decades at least (Fig. 2). Queensland, followed by Western Australia then the Northern Territory has consistently received the most visits from vessels fouled with $P$. viridis, with the estimated number of events approaching 100 annually (Fig. 2). Cumulatively, we estimate there have been thousands of fouled vessels visiting Australian waters over the past 50 years.

These fouled ships are arriving in regions where the habitat is projected to be highly suitable. Indeed, the

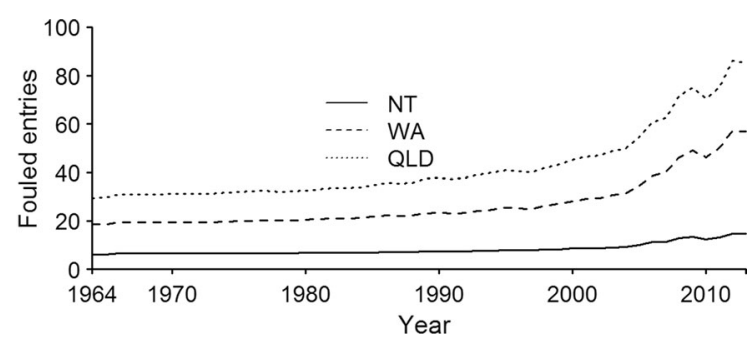

Fig. 2 Estimated number of ships fouled with $P$. viridis entering Western Australia (WA), the Northern Territory (NT) and Queensland (QLD) annually species distribution model based on SST predicts that on the balance of probability, all ports north of $30^{\circ} \mathrm{S}$ latitude are suitable for $P$. viridis - in fact the probabilities would be considered high (Table 1). Averaged over ports within state regions, the mean projected suitabilities are 0.93 for the Northern Territory, 0.85 for Western Australia and 0.83 for Queensland.

\section{Prior distribution on establishment probability $p$}

The probability of establishment fouled vessel entry is difficult to identify. A male and female $P$. viridis must both enter the port undetected, be sexually mature, spawn within a small window, and their progeny settle in the port environment and avoid predation and other challenges to establish. This could well vary according to the time of year. A common default approach to accommodate this uncertain prior knowledge for $p_{i}$ would be to use an uninformed (i.e. "flat") prior on the interval 0-1 [e.g. a Beta $(1.0,1.0)$ distribution]. This is unsatisfactory, however, as there are many forces acting against successful life-cycle completion, hence we have little belief that $p_{i}$ could in fact equal one (implying $100 \%$ of fouled vessel visits to

Table 1 Estimated probability $(\hat{\psi})$ of $P$. viridis suitability for Australian ports of interest calculated using a species distribution model based on sea surface temperature (see Eq. 3 for details)

\begin{tabular}{llllll}
\hline Port & State & $\widehat{\psi}$ & Port & State & $\widehat{\psi}$ \\
\hline Coffs Harbour & NSW & 0.72 & Abbot Point & QLD & 0.94 \\
Yamba & NSW & 0.74 & Brisbane & QLD & 0.74 \\
Darwin & NT & 0.95 & Bundaberg & QLD & 0.76 \\
Gove & NT & 0.91 & Cairns & QLD & 0.86 \\
Groote Eylandt & NT & 0.93 & Gladstone & QLD & 0.77 \\
Broome & WA & 0.92 & Hay Point & QLD & 0.82 \\
Carnarvon & WA & 0.74 & Lucinda & QLD & 0.86 \\
Dampier & WA & 0.89 & Mackay & QLD & 0.82 \\
Derby & WA & 0.92 & Mourilyan & QLD & 0.86 \\
Exmouth & WA & 0.85 & Port Alma & QLD & 0.77 \\
Geraldton & WA & 0.64 & Thursday Isl. & QLD & 0.90 \\
Port Hedland & WA & 0.89 & Townsville & QLD & 0.85 \\
Port Walcott & WA & 0.89 & Weipa & QLD & 0.91 \\
Wyndham & WA & 0.94 & & & \\
\hline
\end{tabular}


suitable habitat will result in establishment). Choosing a $\operatorname{Beta}(1.01,1.01)$, for example, down-weights our prior belief in certain success or failure, but still places equal weight on a high establishment probability. This is also unsatisfactory, however, as in sub-optimal habitats spawning may be strongly seasonal (Vakily 1989), in which case only the corresponding ship visits would have a chance of establishment. After taking these factors into account, we use a $\operatorname{Beta}(2,30)$ distribution as a prior distribution for $p_{i}$. This distribution is heavily weighted towards zero, has a mean value of $c .0 .06$ (i.e. about 1 in 20 visits of a fouled ship will result in establishment) and about $95 \%$ of the probability is less that 0.15 (Fig. 3). Examples of how belief in the value of $p$ decreases with increasing number of failed introductions are shown (Fig. 3).

\section{Updating habitat suitability}

Starting from 1964, the updated (posterior) belief in the habitat suitability of Australian regions of interest for $P$. viridis establishment declines rapidly for both Queensland and Western Australia compared to the Northern Territory (Fig. 4). The difference in the rate of change in belief is driven by the Northern Territory having many fewer fouled vessel arrivals (Fig. 2). By year 2013, the revised belief in the habitat suitability of Western Australian is near-zero (0.14\%), and for the Northern Territory $4.5 \%$ - these are greater than order of magnitude reductions in the belief of suitability. In contrast, following the incursion of $P$. viridis reported in 2001 (Stafford et al. 2007), the estimated habitat suitability for Queensland jumps to one, and by definition remains there (once

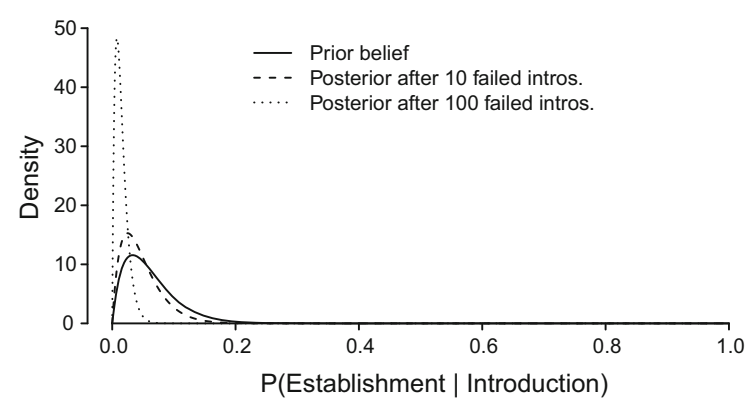

Fig. 3 Posterior (revised) distribution for $p$ after observing $N=$ 10 (dashed line) or $N=100$ (dotted line) visits of fouled vessels with no establishments. The prior value for $p$ is $\operatorname{Beta}(2,30)$. Habitat is assumed suitable

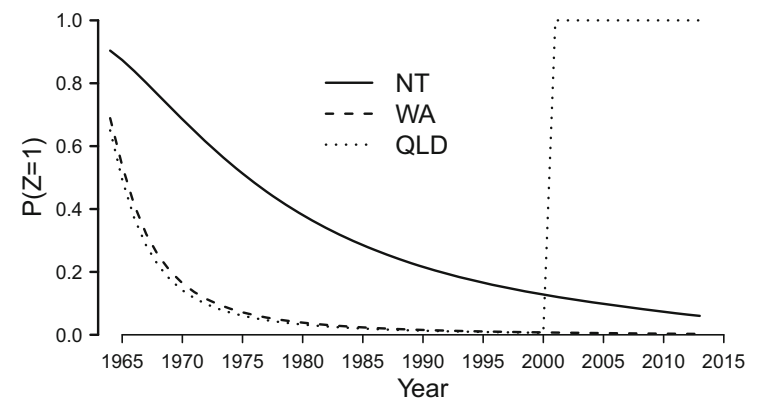

Fig. 4 Changes over time (updates) in the belief in the habitat suitability of state regions for $P$. viridis establishment [i.e. $P(z=1)]$. Note that Queensland (QLD) recorded an incursion of $P$. viridis in 2001

suitable always suitable barring long-term habitat changes). The sense of this is explored further in the Discussion

Updating probability of establishment

Conditional on the habitat being suitable for establishment, the estimated probability of establishment per introduction declines quickly as the cumulative number of fouled vessel visits with no incursions mounts, reaching $0.02 \%$ in Western Australia and $0.4 \%$ in the Northern Territory (Fig. 5). In Queensland, following the recorded incursion of $P$. viridis in 2001 , there is a barely perceptible rise in the estimate for $p$, after which it resumes its downward trajectory in response to further ongoing introductions without establishment, finishing at $0.1 \%$ (Fig. 5).

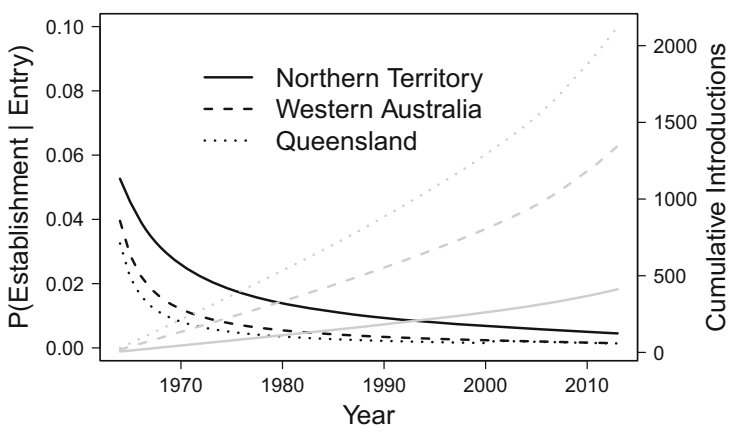

Fig. 5 Progressively updated estimates for the estimated mean probability of establishment per fouled vessel entry of $P$. viridis in ports (left hand axis and black line colour) in response the the cumulative number of introductions (right hand axis and grey line colours) into Western Australian, the Northern Territory and Queensland, assuming the recipient habitat is suitable for establishment 
Yearly unconditional probabilities of $P$. viridis establishment decrease exponentially until about the year 2000 for all state regions (Fig. 6). At this point, the estimated yearly probability of establishment in Queensland undergoes a step change increase in response to the recorded incursion. In addition, the estimated probabilities remains more or less constant (c. $11 \%$ ) despite the accumulation of further failed introductions on account of the increase in the estimated number of fouled arrivals (Fig. 6). For the Northern Territory and Western Australia, yearly probability of establishment drop to $0.4 \%$ and $0.02 \%$, respectively.

\section{Discussion and conclusions}

We have illustrated how prior belief on the ability of an exotic organism to establish (here based on species distribution modelling) can be updated empirically on the basis of knowledge or estimation of failed introductions. In the case of $P$. viridis, despite a high approach rate (also referred to as propagule pressure), the species has failed to establish any self-sustaining populations anywhere in Australia, calling into question the prior expectation of high habitat suitability on the basis of a seas surface temperature based species distribution model. In doing so we've added at quantitative layer to the arguments of Wells (2017). Under the Bayesian paradigm we've updated these beliefs in the ability of a species to invade. Under a hypothetico-deductive approach we would reject the

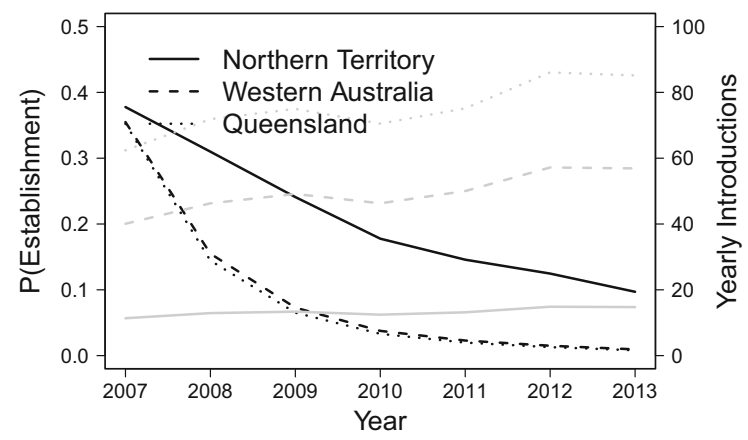

Fig. 6 Progressively updated estimates for the yearly probability of an incursion of $P$. viridis (left hand axis and black line colour) in ports in Western Australian, the Northern Territory and Queensland arising from all fouled vessel entries in each year, using updated estimates of establishment probability (right hand axis and grey line colours) null model as being useful (here the SDM in concert with prior establishment parameters), and seek to find a better one that can withstand the challenge of data.

Our results also highlight the short-comings of modelling purely on a presence-absence basis (ignoring abundance). On the basis of a single incursion (that failed to persist), we have changed our belief entirely regarding the suitability of the Queensland region for $P$. viridis establishment. This is a conservative approach. Note, however, the inability of the population in Trinity Inlet to "take-off" despite considerable opportunity to do so suggests this was a less than optimal environment for $P$. viridis. Given that the impact of invasive species is generally modelled as an increasing function of abundance (e.g. Parker et al. 1999; except for trade-related pests), it makes more sense to explicitly model the abundance of species of concern. Bradley et al. (2018) argue for the importance of explicitly modelling abundance, utilising data sets that record a measure of actual abundance (including zero's), when undertaking risk assessments.

The contradiction between the species distribution model and the apparent lack of establishment of any significant populations could come about in two ways. First, it could simply be that the establishment success per introduction is very small due to hurdles faced by $P$. viridis during the initial colonization phase. An alternative explanation for the lack of establishment success of $P$. viridis could be that some other factor may be rendering the environment unsuitable, thus preventing this species from establishing in Australia. That is, the model being projected is under- or misspecified. Potential unexplored factors include predation, competition, or some other physical or environmental parameter. Further research attempting to resolve the reasons for this contradiction between the species distribution model and the apparent lack of establishment would be appropriate. Guidelines (e.g. Elith and Leathwick 2009) and software Hijmans et al. (2017) are readily available for constructing models, along with guidance to try and build the best model possible. We note, however, that the challenges of projecting models to new environments remain considerable.

After combining information sources, we demonstrate that on a yearly basis, the estimated risk of $P$. viridis establishing in Australia is low and declining. This estimated likelihood will continue to decrease 
into the future in the event of no further populations establishing. Our methods are also applicable to updating invasion beliefs on the basis of deliberate experimental introductions, such as approaches VII (Assessment of species deliberately introduced beyond the current range without environmental manipulation) and VIII (as for VII though with environmental manipulation) of Mack (1996). It was pointed out by a sharp-eyed reviewer that our approach hasn't fully propagated all uncertainty through, particularly in relation to uncertainty in the predictions of original SDM evaluation. The key thing we have demonstrated here is how to update such probabilistic beliefs. More detailed analyses that accommodate for the full uncertainty in prior beliefs are possible.

In conclusion, we have provided estimates of the establishment risk of $P$. viridis in Australia using available data. The results are sensitive to the initial (prior) belief for the probability of establishment arising from a fouled vessel arrival. Strictly speaking, the inference we have presented is restricted to existing ports used in the analysis and the conditions experienced, including the visitation times of vessels. It may not hold for ports in new locations, or existing ports with changed environmental conditions (e.g. through changed land use in river catchments). There is a need for ongoing updating of establishment risk and habitat suitability in the light of additional data on incursions, vessel based fouling rates and the role of other vectors. It remains an open question whether $P$. viridis can be considered a truly invasive marine species in Australian waters. The empirical approach used here indicates this may not be the case based on the evidence to date. Management agencies can use the inference as part of a reassessment of the risks posed by $P$. viridis to the economy and environment.

Acknowledgements Funding was provided by Chevron through the Plant Biosecurity Cooperative Research Centre (Project PBCRC 1106) and the CSIRO Health and Biosecurity business unit. The Australian Defence Science and Technology Organisation provided fouling data on navy ships. Comments of Rieks van Klinken and Petra Kuhnert improved a draft version of the manuscript, that was further improved by the efforts of two anonymous reviewers. We thank them all.

Open Access This article is licensed under a Creative Commons Attribution 4.0 International License, which permits use, sharing, adaptation, distribution and reproduction in any medium or format, as long as you give appropriate credit to the original author(s) and the source, provide a link to the Creative Commons licence, and indicate if changes were made. The images or other third party material in this article are included in the article's Creative Commons licence, unless indicated otherwise in a credit line to the material. If material is not included in the article's Creative Commons licence and your intended use is not permitted by statutory regulation or exceeds the permitted use, you will need to obtain permission directly from the copyright holder. To view a copy of this licence, visit http://creativecommons.org/licenses/by/4.0/.

\section{References}

Baker P, Fajans JS, Arnold WS, Ingrao DA, Marelli DC, Baker SM (2007) Range and dispersal of a tropical marine invader, the Asian green mussel, Perna viridis, in subtropical waters of the southeastern United States. J Shellfish Res 26(2):345-355. https://doi.org/10.2983/0730-8000(2007) 26[345:RADOAT]2.0.CO;2

Barry SC, Hayes KR, Hewitt CL, Behrens HL, Dragsund E, Bakke SM (2008) Ballast water risk assessment: principles, processes, and methods. ICES J Mar Sci J Cons 65(2):121-131. https://doi.org/10.1093/icesjms/fsn004

Beale CM, Lennon JJ (2011) Incorporating uncertainty in predictive species distribution modelling. Philos Trans R Soc B Biol Sci 367(1586):247-258. https://doi.org/10.1098/ rstb.2011.0178

Benson AJ, Marelli DC, Frischer ME, Danforth JM, Williams JD (2001) Establishment of the green mussel, Perna viridis (Linnaeus 1758) (Mollusca: Mytilidae) on the west coast of Florida. J Shellfish Res 20(1):21-29

Blackburn TM, Cassey P, Lockwood JL (2009) The role of species traits in the establishment success of exotic birds. Glob Change Biol 15(12):2852-2860. https://doi.org/10. 1111/j.1365-2486.2008.01841.x

Bradley BA, Allen JM, O’Neill MW, Wallace RD, Bargeron CT, Richburg JA, Stinson K (2018) Invasive species risk assessments need more consistent spatial abundance data. Ecosphere 9(7):e02302. https://doi.org/10.1002/ecs2.2302

Cariton JT, Geller JB (1993) Ecological roulette: the global transport of nonindigenous marine organisms. Science 261(5117):78-82. https://doi.org/10.1126/science.261. 5117.78

Cheong L (1982) Country report, Singapore. In: Bivalve culture in Asia and the Pacific-proceedings of a workshop held in Singapore: 16-19 February, 1982, IDRC (Series), pp 69-71

Elith J, Leathwick JR (2009) Species distribution models: ecological explanation and prediction across space and time. Annu Rev Ecol Evol Syst 40(1):677-697. https://doi.org/ 10.1146/annurev.ecolsys.110308.120159

Elith J, Kearney M, Phillips S (2010) The art of modelling range-shifting species. Methods Ecol Evol 1(4):330-342. https://doi.org/10.1111/j.2041-210X.2010.00036.x

Fitzpatrick MC, Weltzin JF, Sanders NJ, Dunn RR (2007) The biogeography of prediction error: Why does the introduced range of the fire ant over-predict its native range? Glob 
Ecol Biogeogr 16(1):24-33. https://doi.org/10.1111/j. 1466-8238.2006.00258.x

Hayes KR, Sliwa C (2003) Identifying potential marine pests-a deductive approach applied to Australia. Mar Pollut Bull 46(1):91-98. https://doi.org/10.1016/S0025-326X (02)00321-1

Hayes K, Sliwa C, Migus S, McEnnulty F, Dunstan P (2005) National priority pests-part II, ranking of Australian marine pests. Technical report, Department of Environment and Heritage

Hayes KR, McEnnulty F, Sutton C, Cooper S (2008) Ballast water decision support system (DSS) service level agreement (SLA)_part III. Technical report, Australian Government Department of Agriculture, Fisheries and Forestry

Hewitt CL, Campbell ML, Thresher RE, Martin RB, Boyd S, Cohen BF, Currie DR, Gomon MF, Keough MJ, Lewis JA et al (2004) Introduced and cryptogenic species in Port Phillip Bay, Victoria, Australia. Mar Biol 144(1):183-202. https://doi.org/10.1007/s00227-003-1173-x

Hijmans RJ, Phillips S, Leathwick J, Elith J (2017) Package 'dismo'. http://rspatial.org/sdm/

Krebs CJ (2009) The experimental analysis of distribution and abundance, 6th edn. Benjamin Cummings, San Francisco

Lockwood JL, Cassey P, Blackburn TM (2009) The more you introduce the more you get: the role of colonization pressure and propagule pressure in invasion ecology. Divers Distrib 15(5):904-910. https://doi.org/10.1111/j.14724642.2009.00594.x

Mack RN (1996) Predicting the identity and fate of plant invaders: emergent and emerging approaches. Biol Invasions 78:107-121. https://doi.org/10.1016/00063207(96)00021-3

NIMPIS (2014) Perna viridis impacts and vectors. National Introduced Marine Pest Information System. https://www. marinepests.gov.au/pests/nimpis. Accessed 25 Nov 2019

Paini DR, Mwebaze P, Kuhnert PM, Kriticos DJ (2018) Global establishment threat from a major forest pest via international shipping: Lymantria dispar. Sci Rep 8(1):13723. https://doi.org/10.1038/s41598-018-31871-y

Parker IM, Simberloff D, Lonsdale WM, Goodell K, Wonham M, Kareiva PM, Williamson MH, Von Holle B, Moyle PB, Byers JE, Goldwasser L (1999) Impact: toward a framework for understanding the ecological effects of invaders. Biol Invasions 1(1):3-19. https://doi.org/10. 1023/a:1010034312781

R Core Team (2018) R: a language and environment for statistical computing. http://www.R-project.org/

Seebens H, Blackburn TM, Dyer EE, Genovesi P, Hulme PE, Jeschke JM, Pagad S, Pysek P, Winter M, Arianoutsou M, Bacher S, Blasius B, Brundu G, Capinha C, Celesti-Grapow L, Dawson W, Dullinger S, Fuentes N, Joger H, Kartesz J, Kenis M, Kreft H, Kuhn I, Lenzner B, Liebhold A, Mosena A, Moser D, Nishino M, Pearman D, Pergl J, Rabitsch W, Rojas-Sandoval J, Roques A, Rorke S, Rossinelli S, Roy HE, Scalera R, Schindler S, Stajerova K, Tokarska-Guzik B, van Kleunen M, Walker K, Weigelt P, Yamanaka T, Essl F (2017) No saturation in the accumulation of alien species worldwide. Nat Commun 8(14):435. https://doi.org/10.1038/ncomms14435

Sivalingam P (1977) Aquaculture of the green mussel, Mytilus viridis Linnaeus, in Malaysia. Aquaculture 11:297-312

Stafford H, Willan RC, Neil KM et al (2007) The invasive Asian Green Mussel, Perna viridis (Linnaeus, 1758) (Bivalvia: Mytilidae), breeds in Trinity Inlet, tropical northern Australia. Molluscan Res 27(2):105-109

Summerson R, Darbyshire R, Lawrence E (2007) Invasive marine species range mapping. Technical report, Australian Government Bureau of Rural Sciences

Thresher RE (1999) Diversity, impacts and options for managing invasive marine species in Australian waters. Aust J Environ Manag 6(3):137-148. https://doi.org/10.1080/ 14486563.1999.10648462

Vakily J (1989) The biology and culture of mussels of the genus Perna. ICLARM Contribution, International Center for Living Aquatic Resources Management

Wells FE (2017) If the Asian green mussel, Perna viridis (Linnaeus, 1758), poses the greatest invasive marine species threat to Australia, why has it not invaded? Molluscan Res 37(3):167-174. https://doi.org/10.1080/13235818. 2017.1322676

Publisher's Note Springer Nature remains neutral with regard to jurisdictional claims in published maps and institutional affiliations. 